\title{
$k$-GENERALIZED FIBONACCI NUMBERS WHICH ARE CONCATENATIONS OF TWO REPDIGITS
}

\author{
Adel Alahmadi, Alaa Altassan, Florian luca and Hatoon Shoaib \\ King Abdulaziz University, Saudi Arabia, Wits University, South Africa and \\ UNAM, Mexico
}

\begin{abstract}
We show that the $k$-generalized Fibonacci numbers that are concatenations of two repdigits have at most four digits.
\end{abstract}

\section{INTRODUCTION}

Let $g \geq 2$ be an integer. A natural number $N$ is called a base $g$-repdigit if all of its base $g$-digits are equal; that is, if

$$
N=a\left(\frac{g^{m}-1}{g-1}\right), \quad \text { for some } \quad m \geq 1 \text { and } a \in\{1,2, \ldots, g-1\} .
$$

When $g=10$, we omit the base and we simply say that $N$ is a repdigit. Diophantine equations involving repdigits were considered in several recent papers in which their authors found all repdigits that are perfect powers, or Fibonacci numbers, or generalized Fibonacci numbers, and so on (see $[2,5,8$, $15,16,17,18,20]$ for a sample of such results).

Given positive integers $A_{1}, \ldots, A_{t}$, we write

$$
\overline{A_{1} \cdots A_{t}(g)}
$$

for the concatenation of their base $g$ strings of digits. We omit writing $g$ when $g=10$. Thus, the repdigit $N$ shown at (1.1) is just

$$
N=\underbrace{\overline{a \cdots a}}_{m \text { times }}(g) \text {, }
$$

2020 Mathematics Subject Classification. 11A25, 11B39, 11J86.

Key words and phrases. $k$-Fibonacci numbers, applications of Baker's method, digits.

This study was funded by King Abdulaziz University, Deanship of Scientific Research (grant number RG-26-130-40). 
whereas the concatenation of two repdigits in base 10 is

$$
\overline{a \cdots a b \cdots b}, \quad \text { where } \quad a, b \in\{0,1, \ldots, 9\} \text {. }
$$

We impose that $a \neq 0$. That is, we do not allow the leading digit to be zero, but the final digit might be zero. Let $\left\{F_{m}\right\}_{m \geq 0}$ be the Fibonacci sequence given by

$$
F_{m+2}=F_{m+1}+F_{m}, \quad \text { for all } m \geq 0,
$$

where $F_{0}=0$ and $F_{1}=1$. The first few terms of this sequence are

$$
0,1,1,2,3,5,8,13,21,34,55,89,144,233,377,610,987 \text {. }
$$

In 2011, S. Díaz-Alvarado and F. Luca ([2]) determined all Fibonacci numbers that are sums of two repdigits. In [3], Banks and Luca considered Diophantine equations with concatenations of members of binary recurrences. For example, they showed that the only Fibonacci numbers which are concatenations of two other Fibonacci numbers are 13, 21,55. In [1], we studied Fibonacci numbers which are concatenations of two nonzero repdigits. The largest example is $F_{14}=377$. In this paper, we extend our result from [1] and study $k$-generalized Fibonacci numbers which are concatenations of two repdigits. Recall that the $k$-generalized Fibonacci sequence $\left\{F_{n}^{(k)}\right\}_{n \geq-(k-2)}$ is given by the recurrence $F_{n+k}^{(k)}=F_{n+k-1}^{(k)}+\cdots+F_{n}^{(k)}$ for all $n \geq-(k-2)$ with initial values $0,0, \ldots, 0,1$ (with $k-1$ consecutive zeros), where the value of 1 corresponds to $n=1$; namely, $F_{1}^{(k)}=1$, and the remaining zeros are in the past $F_{j}^{(k)}=0$ for $j \in\{-(k-2),-(k-3), \ldots, 0\}$. When $k=2$ this coincides with the Fibonacci sequence. Obviously, every number with at most two digits is a concatenation of two repdigits. Thus, we only look at the case of at least three digits. Our result is the following theorem.

THEOREM 1.1. The only k-generalized Fibonacci numbers with at least three digits which are concatenations of two repdigits are

$$
\begin{aligned}
& F_{12}^{(2)}=144 ; \quad F_{13}^{(2)}=233 ; \quad F_{14}^{(2)}=377 ; \quad F_{12}^{(4)}=773 \\
& F_{13}^{(7)}=2000 ; \quad F_{10}^{(8)}=255 ; \quad F_{11}^{(9)}=511 .
\end{aligned}
$$

We organise this paper as follows. In Section 2, we recall some elementary properties of $k$-generalized Fibonacci numbers, a result due to Matveev on lower bounds for nonzero linear forms of logarithms of algebraic numbers, and a result on the Baker-Davenport reduction. We also need an elementary result about powers of two which are concatenations of two repdigits. The proof of Theorem 1.1 is done in Section 3. 


\section{Preliminaries}

In this section, we collect some facts about algebraic number theory and the theory of linear forms in logarithms of algebraic numbers and $k$-generalized Fibonacci numbers.

2.1. Linear forms in logarithms. We start with some notations and terminology from algebraic number theory.

Let $\eta$ be an algebraic number of degree $d$ with minimal primitive polynomial over the integers

$$
a_{0} x^{d}+a_{1} x^{d-1}+\cdots+a_{d}=a_{0} \prod_{i=1}^{d}\left(x-\eta^{(i)}\right),
$$

where the leading coefficient $a_{0}$ is positive and the $\eta^{(i)} \mathrm{s}$ are the conjugates of $\eta$. Then the logarithmic height of $\eta$ is given by

$$
h(\eta):=\frac{1}{d}\left(\log a_{0}+\sum_{i=1}^{d} \log \left(\max \left\{\left|\eta^{(i)}\right|, 1\right\}\right)\right) .
$$

In particular, if $\eta=p / q$ is a rational number with $\operatorname{gcd}(p, q)=1$ and $q \geq 1$, then $h(\eta)=\log \max \{|p|, q\}$. The following are some of the properties of the logarithmic height function $h(\cdot)$, which will be used in the next section of this paper without reference:

$$
\begin{aligned}
h\left(\eta \gamma^{ \pm 1}\right) & \leq h(\eta)+h(\gamma), \\
h(\eta+\gamma) & \leq h(\eta)+h(\gamma)+\log 2, \\
h\left(\eta^{s}\right) & =|s| h(\eta) \quad(s \in \mathbb{Z}) .
\end{aligned}
$$

In order to prove our main result Theorem 1.1, we need to use a couple of times a Baker-type lower bound for a nonzero linear form in logarithms of algebraic numbers. There are many such bounds the literature like that of Baker and Wüstholz from [4]. We use the one of Matveev from [19]. Matveev proved the following theorem, which is one of our main tools in this paper.

Theorem 2.1 (Matveev's theorem). Let $\gamma_{1}, \ldots, \gamma_{t}$ be positive real algebraic numbers in a real algebraic number field $\mathbb{K}$ of degree $D, b_{1}, \ldots, b_{t}$ be nonzero integers, and assume that

$$
\Lambda:=\gamma_{1}^{b_{1}} \cdots \gamma_{t}^{b_{t}}-1,
$$

is nonzero. Then

$$
\log |\Lambda|>-1.4 \times 30^{t+3} \times t^{4.5} \times D^{2}(1+\log D)(1+\log B) A_{1} \cdots A_{t},
$$

where

$$
B \geq \max \left\{\left|b_{1}\right|, \ldots,\left|b_{t}\right|\right\}
$$

and

$$
A_{i} \geq \max \left\{D h\left(\gamma_{i}\right),\left|\log \gamma_{i}\right|, 0.16\right\}, \quad \text { for all } \quad i=1, \ldots, t .
$$


2.2. The Baker-Davenport reduction method. During the course of our calculations, we get some upper bounds on our variables which are too large, thus we need to reduce them. To do so, we use some results from the theory of continued fractions. Specifically, for a nonhomogeneous linear form in two integer variables, we use a slight variation of a result due to Dujella and Pethő (see [11, Lemma 5a]). For a real number $X$, we write $\|X\|:=\min \{|X-n|$ : $n \in \mathbb{Z}\}$ for the distance from $X$ to the nearest integer.

Lemma 2.2. Let $M$ be a positive integer, $p / q$ be a convergent of the continued fraction of the irrational number $\tau$ such that $q>6 M$, and $A, B, \mu$ be real numbers with $A>0$ and $B>1$. If $\varepsilon:=\|\mu q\|-M\|\tau q\|>0$, then there is no solution to the inequality

$$
0<|u \tau-v+\mu|<A B^{-w},
$$

in positive integers $u, v$ and a real number $w$ with

$$
u \leq M \quad \text { and } \quad w \geq \frac{\log (A q / \varepsilon)}{\log B} .
$$

The above lemma cannot be applied when $\mu=0$ (since then $\varepsilon<0$ ). In this case, we use the following criterion of Legendre (see [12, Theorem 8.2.4 and top of page 287]).

LEMMA 2.3 (Legendre). Let $\tau$ be a real number and $x, y$ be integers such that

$$
\left|\tau-\frac{x}{y}\right|<\frac{1}{2 y^{2}}
$$

Then $x / y=p_{k} / q_{k}$ is a convergent of $\tau$. Furthermore,

$$
\left|\tau-\frac{x}{y}\right| \geq \frac{1}{\left(a_{k+1}+2\right) y^{2}}
$$

2.3. $k$-generalized Fibonacci numbers. One checks easily that the first $k+1$ nonzero terms in $F_{n}^{(k)}$ are powers of 2, namely

$$
F_{1}^{(k)}=1, \quad F_{2}^{(k)}=1, \quad F_{3}^{(k)}=2, \quad F_{4}^{(k)}=4, \ldots, F_{k+1}^{(k)}=2^{k-1} .
$$

The next term is $F_{k+2}^{(k)}=2^{k}-1$. It is known that the characteristic polynomial of the $k$-generalized Fibonacci numbers $F^{(k)}:=\left\{F_{n}^{(k)}\right\}_{n \geq-(k-2)}$, namely

$$
\Psi_{k}(x):=x^{k}-x^{k-1}-\cdots-x-1,
$$

is irreducible over $\mathbb{Q}[x]$ and has just one root outside the unit circle. Let $\alpha:=\alpha(k)$ denote the single real root larger than 1 , which is located between $2\left(1-2^{-k}\right)$ and 2 (see [10]). This is called the dominant root of $F^{(k)}$. To simplify notation, in our application we shall omit the dependence of $\alpha$ on $k$. We shall use $\alpha^{(1)}, \ldots, \alpha^{(k)}$ for all roots of $\Psi_{k}(x)$ with the convention that $\alpha:=\alpha^{(1)}$. 
We now consider, for an integer $k \geq 2$, the function

$$
f_{k}(z)=\frac{z-1}{2+(k+1)(z-2)} \quad \text { for } \quad z \in \mathbb{C} .
$$

With this notation, Dresden and Du presented in [10] the following "Binetlike" formula for the terms of $F^{(k)}$ :

$$
F_{n}^{(k)}=\sum_{i=1}^{k} f_{k}\left(\alpha^{(i)}\right)\left(\alpha^{(i)}\right)^{n-1}
$$

It was proved in [10] that the contribution of the roots which are inside the unit circle to the formula (2.6) is very small, namely that the approximation

$$
\left|F_{n}^{(k)}-f_{k}(\alpha) \alpha^{n-1}\right|<\frac{1}{2} \text { holds for all } n \geq-(k-2) .
$$

It was proved by Bravo and Luca in [5] that

$$
\alpha^{n-2} \leq F_{n}^{(k)} \leq \alpha^{n-1} \quad \text { holds for } n \geq 1 \text { and } k \geq 2 .
$$

We next present some useful lemmas that will be used in the next section of this paper. The following lemma was proved by Bravo and Luca in [5].

LEMMA 2.4. Let $k \geq 2$, $\alpha$ be the dominant root of $\left\{F_{n}^{(k)}\right\}_{n \geq(k-2)}$, and consider the function $f_{k}(z)$ defined in (2.5).

(i) The inequalities

$$
\frac{1}{2}<f_{k}(\alpha)<\frac{3}{4}, \quad \text { and } \quad\left|f_{k}\left(\alpha^{(i)}\right)\right|<1, \quad 2 \leq i \leq k
$$

hold.

(ii) The logarithmic height of $f_{k}(\alpha)$ satisfies $h\left(f_{k}(\alpha)\right)<3 \log k$.

The following lemma was proved in [9].

LEMMA 2.5. For $1 \leq n<2^{k / 2}$ and $k \geq 10$, we have

$$
F_{n}^{(k)}=2^{n-2}(1+\zeta), \text { where }|\zeta|<\frac{5}{2^{k / 2}} .
$$

Finally, the following lemma is [14, Lemma 7].

LEMMA 2.6. If $m \geq 1, T \geq\left(4 m^{2}\right)^{m}$ and

$$
\frac{x}{(\log x)^{m}}<T, \quad \text { then } \quad x<2^{m} T(\log T)^{m} .
$$


2.4. Powers of two which are concatenations of two repdigits. In this subsection we prove the following lemma.

LEMMA 2.7. There are no powers of two with more than two digits which are concatenations of two repdigits.

Proof. Write

$$
2^{n}=\overline{\underbrace{a \cdots a}_{m \text { times }} \underbrace{b \cdots b}_{\text {times }}}, \quad \text { where } \quad a, b \in\{0,1, \ldots, 9\}, \quad a \neq 0 .
$$

This can be rewritten as

$$
9 \times 2^{n}=a\left(10^{m}-1\right) 10^{\ell}+b\left(10^{\ell}-1\right) .
$$

Note that $b$ cannot be zero since otherwise $9 \times 2^{n}$ would be a multiple of 5 . Thus, $b \in\{1, \ldots, 9\}$. In particular, $\nu_{2}\left(b\left(10^{\ell}-1\right)\right) \leq 3$, where for an integer $m$ we write $\nu_{2}(m)$ for the exponent of 2 in the factorization of $m$. In particular, if $n \geq 4$, then $\ell \leq 3$. In this case,

$$
9 \times 2^{n}=a 10^{m+\ell}+\left((b-a) 10^{\ell}-b\right) .
$$

The integer $(b-a) 10^{\ell}-b$ is not zero (otherwise $9 \times 2^{n}$ would be divisible by 5 ), and it is in absolute value at most $9 \times 10^{3}+9<2^{14}$. In particular, if $n \geq 14$, then $m+\ell \leq 13$. Thus, in this last case, we get

$$
2^{n}<10^{13}+10^{3}+1<2^{44}
$$

so $n \leq 43$. A numerical check finishes the proof.

\section{The Proof of Theorem 1.1}

We work with the equation

$$
F_{n}^{(k)}=\overline{\underbrace{a \cdots a}_{m \text { times }} \underbrace{b \cdots b}_{\text {times }}}, \quad \text { where } \quad a, b \in\{0,1, \ldots, 9\}, \quad a \neq 0 .
$$

3.1. The low range. We ignore the repdigit case (namely, the case $a=b$ or $a=0$ in equation (3.1)) since that has been treated in [5]. We next check the case $n \leq 1000$. It suffices to also assume that $k \leq 1000$, since if $k>1000$, then $n<k$, so $F_{n}^{(k)}$ is a power of 2 , and we know that in this case equation (3.1) has no solutions with $m+\ell \geq 3$ by Lemma 2.7. Then $F_{n}^{(k)} \leq 2^{n-1} \leq 2^{999}$ has at most 300 digits. We generated the list of all $F_{n}^{(k)}$ for $2 \leq k, n \leq 1000$ as well as the list of all numbers which are concatenations of two repdigits and with a total of at most 300 digits and intersected these two lists obtaining the examples mentioned in the theorem. From now on, we assume that $n>1000$. 
3.2. Bounding $n$ in terms of $k$. We exploit (3.1). That is

$$
\begin{aligned}
F_{n}^{(k)} & =\overline{\underbrace{a \cdots a}_{m \text { times }} \underbrace{b \cdots b}_{\text {times }}} \\
& =\overline{\underbrace{\overline{a \cdots a}}_{m \text { times }} \times 10^{\ell}+\underbrace{b \cdots b}_{\ell \text { times }}} \\
& =\frac{1}{9}\left(a 10^{m+\ell}-(a-b) 10^{\ell}-b\right) .
\end{aligned}
$$

We next comment on the size of $n$ versus $m+\ell$.

Lemma 3.1. All solutions of equation (3.1) satisfy

$$
(m+\ell) \log 10-2 \leq n \log \alpha<(m+\ell) \log 10+2 .
$$

Proof. The proof follows easily from the fact that $\alpha^{n-2}<F_{n}^{(k)}<\alpha^{n-1}$. By estimate (2.8), one can see that

$$
\alpha^{n-2}<F_{n}^{(k)}<10^{m+\ell} .
$$

Taking the logarithm of both sides, we get $(n-2) \log \alpha<(m+\ell) \log 10$, which leads to

$$
n \log \alpha<(m+\ell) \log 10+2 \log \alpha<(m+\ell) \log 10+2 .
$$

The lower bound follows similarly from the bound $10^{m+\ell-1} \leq F_{n}^{(k)}<\alpha^{n-1}$.

We next examine (3.2) in two different steps as follows.

Step 1. Equation (3.2), the Binet formula (2.6) for $F_{n}^{(k)}$ and approximation (2.7), give

$$
9 f_{k}(\alpha) \alpha^{n-1}-a 10^{m+\ell}=9 \zeta_{n}-\left((a-b) 10^{\ell}+b\right), \quad\left|\zeta_{n}\right|<1 / 2 .
$$

Here, $\zeta_{n}:=f_{k}(\alpha) \alpha^{n-1}-F_{n}^{(k)}$. From the above, we deduce that

$$
\begin{aligned}
\left|9 f_{k}(\alpha) \alpha^{n-1}-a 10^{m+\ell}\right| & =\left|9 \zeta_{n}-\left((a-b) 10^{\ell}+b\right)\right| \\
& \leq\left(9 \cdot 10^{\ell}+9\right)+9 / 2 \\
& <15 \times 10^{\ell} .
\end{aligned}
$$

Thus, dividing both sides by $a 10^{m+\ell}$ we get

$$
\left|\left(\frac{9 f_{k}(\alpha)}{a}\right) \alpha^{n-1} 10^{-m-\ell}-1\right|<\frac{15 \times 10^{\ell}}{a 10^{m+\ell}}<\frac{15}{10^{m}} .
$$

Let

$$
\Gamma_{1}:=\left(\frac{9 f_{k}(\alpha)}{a}\right) \alpha^{n-1} 10^{-m-\ell}-1
$$


We compare this upper bound with the lower bound on the quantity $\Gamma_{1}$ given by Theorem 2.1. Observe first that $\Gamma_{1}$ is not zero, for if it were, then

$$
\frac{9 f_{k}(\alpha)}{a}=\frac{10^{m+\ell}}{\alpha^{n-1}}
$$

Conjugating the above relation with some non-trivial Galois automorphism $\sigma$ of $\mathbb{Q}[\alpha]$ which maps $\alpha$ to $\alpha^{(i)}$ for some $i=2, \ldots, k$, we get

$$
\frac{9 f_{k}\left(\alpha^{(i)}\right)}{a}=\frac{10^{m+\ell}}{\left(\alpha^{(i)}\right)^{n-1}} .
$$

Taking absolute values and using the estimate from Lemma 2.4 (i) as well as the fact that $\left|\alpha^{(i)}\right|<1$ for $i=2, \ldots, k$, we get

$$
9>\frac{\left|9 f_{k}\left(\alpha^{(i)}\right)\right|}{a}=\frac{10^{m+\ell}}{\left|\alpha^{(i)}\right|^{n-1}}>10^{m+\ell},
$$

a contradiction.

With the notation of Theorem 2.1, we take $t:=3$ and

$$
\gamma_{1}:=\frac{9 f_{k}(\alpha)}{a}, \gamma_{2}:=\alpha, \gamma_{3}:=10, b_{1}:=1, b_{2}:=n-1, b_{3}:=-m-\ell .
$$

Since $10^{m+\ell-1}<F_{n}^{(k)}<\alpha^{n-1}$, we have that $m+\ell \leq n$. So, we take $B:=n$. Observe that $\mathbb{L}:=\mathbb{Q}\left(\eta_{1}, \eta_{2}, \eta_{3}\right)=\mathbb{Q}(\alpha)$, so $D=k$. We note that $h\left(\gamma_{2}\right) \leq 1 / k$, $h\left(\gamma_{3}\right)=\log 10$ and, by Lemma 2.4 (ii) and the properties of the heights,

$$
h\left(\gamma_{1}\right) \leq h(9 / a)+h\left(f_{k}(\alpha)\right) \leq \log 9+3 \log k \leq 7 \log k \text { for all } k \geq 2 .
$$

Thus, we can take

$$
A_{1}:=7 k \log k, \quad A_{2}:=1, \quad A_{3}:=k \log 10 .
$$

Theorem 2.1 tells us that

$$
\begin{aligned}
\log \left|\Gamma_{1}\right| & >-1.4 \cdot 30^{6} 3^{4.5} k^{2}(1+\log k)(1+\log n)(7 k \log k)(k \log 10) \\
& >-5.8 \times 10^{12} k^{4}(\log k)^{2}(1+\log n) .
\end{aligned}
$$

In the above, we used the inequality $1+\log k \leq 2.5 \log k$ which holds for all $k \geq 2$. Comparing this last inequality with (3.3) leads to

$$
m \log 10-\log 15<5.8 \cdot 10^{12} k^{4}(\log k)^{2}(1+\log n)
$$

giving

$$
m \log 10<5.9 \cdot 10^{12} k^{4}(\log k)^{2}(1+\log n) .
$$


STEP 2. Equation (3.2) can also be rewritten as

$$
f_{k}(\alpha) \alpha^{n-1}-\left(\frac{a 10^{m}-(a-b)}{9}\right) 10^{\ell}=\zeta_{n}-\frac{b}{9}
$$

which gives

$$
\left|f_{k}(\alpha) \alpha^{n-1}-\left(\frac{a 10^{m}-(a-b)}{9}\right) 10^{\ell}\right|=\left|\zeta_{n}-\frac{b}{9}\right|<1.5 .
$$

Thus, dividing both sides by $f_{k}(\alpha) \alpha^{n-1}$, we get

$$
\left|\left(\frac{a 10^{m}-(a-b)}{9 f_{k}(\alpha)}\right) \alpha^{-n+1} 10^{\ell}-1\right|<\frac{1.5}{f_{k}(\alpha) \alpha^{n-1}}<\frac{3}{\alpha^{n-1}},
$$

where for the last inequality above we used that $f_{k}(\alpha)>1 / 2$ (see Lemma 2.4). Put

$$
\Gamma_{2}:=\left(\frac{a 10^{m}-(a-b)}{9 f_{k}(\alpha)}\right) \alpha^{-n+1} 10^{\ell}-1 .
$$

Assume first that $\Gamma_{2}=0$. We then get

$$
\frac{a 10^{m}-(a-b)}{9 f_{k}(\alpha)}=\frac{\alpha^{n-1}}{10^{\ell}} .
$$

Conjugating the above relation by any authomorphism of $\mathbb{K}$ which sends $\alpha$ to $\alpha^{(i)}$ for some $i \geq 2$, and taking absolute values, we get

$$
\frac{a 10^{m}-(a-b)}{9\left|f_{k}\left(\alpha^{(i)}\right)\right|}=\frac{\left|\alpha^{(i)}\right|^{n-1}}{10^{\ell}} \text {. }
$$

Dividing relations (3.8) and (3.9) and using Lemma 2.4 (i), we get

$$
1>\left|f_{k}\left(\alpha^{(i)}\right) \| \alpha^{(i)}\right|^{n-1}=f_{k}(\alpha) \alpha^{n-1}>\frac{1}{2}\left(\frac{1+\sqrt{5}}{2}\right)^{n-1},
$$

a contradiction since $n>1000$.

Thus, $\Gamma_{2} \neq 0$. With the notation of Theorem 2.1, we take $t:=3$,

$$
\gamma_{1}:=\left(\frac{a 10^{m}-(a-b)}{9 f_{k}(\alpha)}\right), \gamma_{2}:=\alpha, \gamma_{3}:=10, b_{1}:=1, b_{2}:=-n+1, b_{3}:=\ell
$$

Observe that $\gamma_{1} \neq 0$, otherwise inequality (3.6) implies that $\alpha^{n-1}<3$, which is false for $n>1000$. As mentioned before $\ell<n$, therefore we can take 
$B:=n$. Furthermore, we have

$$
\begin{aligned}
h\left(\gamma_{1}\right) & =h\left(\frac{a 10^{m}-(a-b)}{9 f_{k}(\alpha)}\right) \\
& \leq h\left(9 f_{k}(\alpha)\right)+h\left(a 10^{m}-(a-b)\right) \\
& \leq \log 9+h\left(f_{k}(\alpha)\right)+h\left(a 10^{m}\right)+h(a-b)+\log 2 \\
& \leq 3 \log 9+\log 2+m \log 10+3 \log k \\
& \leq 5.9 \cdot 10^{12} k^{4}(\log k)^{2}(1+\log n)+3 \log 9+\log 2+3 \log k \\
& <6 \cdot 10^{12} k^{4}(\log k)^{2}(1+\log n),
\end{aligned}
$$

where in the above string of inequalities we used the properties of heights (2.1) and the bound (3.5). Thus, we can take

$$
A_{1}:=6 \cdot 10^{12} k^{5}(\log k)^{2}(1+\log n), \quad A_{2}:=1, \quad A_{3}:=k \log 10 .
$$

Theorem 2.1 tells us that:

$$
\begin{aligned}
\log \left|\Gamma_{2}\right| & >-1.4 \cdot 30^{6} 3^{4.5} k^{2}(1+\log k)(1+\log n)(k \log 10) A_{1} \\
& >-10^{12} k^{3}(\log k)(1+\log n) A_{1} \\
& >-6 \cdot 10^{24} k^{8}(\log k)^{3}(1+\log n)^{2} .
\end{aligned}
$$

Comparing this last inequality with (3.6) we get

$$
(n-1) \log \alpha-\log 3<6 \cdot 10^{24} k^{8}(\log k)^{3}(1+\log n)^{2} .
$$

The above inequality together with the fact that $\alpha \geq(1+\sqrt{5}) / 2$ gives us

$$
n<1.3 \times 10^{25} k^{8}(\log k)^{3}(1+\log n)^{2} .
$$

We need a bound on $n$ which does not involve $\log n$ in the right-hand side. We may assume that $n>10^{10}$. In this case, $1.3(1+\log n)^{2}<2(\log n)^{2}$, so we get

$$
n<2 \times 10^{25} k^{8}(\log k)^{3}(\log n)^{2} .
$$

Thus,

$$
\frac{n}{(\log n)^{2}}<2 \times 10^{25} k^{8}(\log k)^{3}:=T .
$$

We apply Lemma 2.6 with $m:=2$ and the above $T$ noting that it certainly satisfies the lower bound $T \geq\left(4 m^{2}\right)^{m}=2^{8}$. We thus get

$$
\begin{aligned}
n<4 T(\log T)^{2} & \leq 8 \times 10^{25} k^{8}(\log k)^{3}\left(\log \left(2 \times 10^{25}\right)+11 \log k\right)^{2} \\
& \leq 8 \times 10^{25} k^{8}(\log k)^{3}(11 \log k)^{2}\left(\frac{\log \left(2 \times 10^{25}\right)}{11 \log 2}+1\right)^{2} \\
& <8 \times 10^{29} k^{8}(\log k)^{5} .
\end{aligned}
$$

Lemma 3.1 together with the fact that $\alpha<2$ implies

$$
m+\ell<\frac{1}{\log 10}\left(8 \times 10^{29} k^{8}(\log k)^{5} \log 2+2\right)<4 \times 10^{29} k^{8}(\log k)^{5} .
$$


We summarise what we have proved so far in the following lemma.

LEMMA 3.2. All solutions of equation (3.1) satisfy

$$
m+\ell<4 \times 10^{29} k^{8}(\log k)^{5} \quad \text { and } \quad n<8 \times 10^{29} k^{8}(\log k)^{5} .
$$

3.3. An absolute bound on $n$ when $k$ is large. We want to apply Lemma 2.5. For this, we need that $n<2^{k / 2}$. This is satisfied, by Lemma 3.2, provided

$$
8 \times 10^{29} k^{8}(\log k)^{5}<2^{k / 2},
$$

and this last inequality holds for $k \geq 400$. So, assuming $k \geq 400$, we can write

$$
F_{n}^{(k)}=2^{n-2}(1+\zeta), \quad|\zeta|<\frac{5}{2^{k / 2}} .
$$

Since $k$ is large, it follows that $|\zeta|<1 / 2$. Thus, $1+\zeta \in(1 / 2,3 / 2)$, so in particular $2^{n-2} \in\left[(2 / 3) F_{n}^{(k)} / 2,2 F_{n}^{(k)}\right]$. Thus, $2^{n-2}>(2 / 3) F_{n}^{(k)} \geq(2 / 3) 10^{m+\ell-1}$. Substituting (3.11) into (3.2), we get

$$
2^{n-2}(1+\zeta)=\frac{1}{9}\left(a 10^{m+\ell}-(a-b) 10^{\ell}-b\right),
$$

which can be rewritten as

$$
\left|2^{n-2}-(a / 9) 10^{m+\ell}\right| \leq 2^{n-2}|\zeta|+\frac{|a-b| 10^{\ell}+b}{9} \leq \frac{5 \times 2^{n-2}}{2^{k / 2}}+1.1 \times 10^{\ell} .
$$

Thus,

$$
\begin{aligned}
\left|(a / 9) 10^{m+\ell} 2^{-(n-2)}-1\right| & \leq \frac{5}{2^{k / 2}}+\frac{1.1 \times 10^{\ell}}{2^{n-2}} \\
& \leq \frac{5}{2^{k / 2}}+\frac{1.1 \times 1.5}{10^{m-1}} \\
& \leq 22 \max \left\{\frac{1}{2^{k / 2}}, \frac{1}{10^{m}}\right\} .
\end{aligned}
$$

We take

$$
\Gamma_{3}:=(a / 9) 10^{m+\ell} 2^{-(n-2)}-1 .
$$

We now apply Matveev's Theorem 2.1 to $\Gamma_{3}$ defined above. It is easy to see that $\Gamma_{3} \neq 0$, for if $\Gamma_{3}$ were zero, then $10^{m+\ell} a=9 \cdot 2^{n-2}$, which is impossible because the left-hand side above is a multiple of 5 and the right-hand side is not. So, we take $t:=3$,

$$
\left(\gamma_{1}, \gamma_{2}, \gamma_{3}, b_{1}, b_{2}, b_{3}\right):=(a / 9,10,2,1, m+\ell,-(n-2)) .
$$

We have $\mathbb{L}=\mathbb{Q}$, so $D:=1, A_{1}:=\log 9 \geq h\left(\gamma_{1}\right), A_{2}:=\log 10, A_{3}:=\log 2$. Further, we may take $B:=n$ as in the estimates for $\Gamma_{2}$. Thus,

$$
\log \left|\Gamma_{3}\right|>-6 \times 10^{11}(1+\log n) .
$$

Thus, we get

$$
\min \{(k / 2) \log 2, m \log 10\}<6 \times 10^{11}(1+\log n)+\log 22<10^{12} \log n,
$$


where the last inequality holds since $n>1000$. Assume first that

$$
(k / 2) \log 2=\min \{(k / 2) \log 2, m \log 10\} .
$$

We then get

$$
k<\frac{2}{\log 2}\left(10^{12} \log n\right)<3 \times 10^{12} \log n .
$$

Inserting this into the bound on $n$ in terms of $k$ of Lemma 3.2 and using the fact that $n \geq k$ (otherwise $F_{n}^{(k)}$ is a power of 2 which is not a concatenation of two repdigits by Lemma 2.7), we get

$$
\begin{aligned}
n & <8 \times 10^{29} k^{8}(\log k)^{5}<8 \times 10^{29} k^{8}(\log n)^{5} \\
& <8 \times 10^{29}\left(3 \times 10^{12}\right)^{8}(\log n)^{13}<6 \times 10^{129}(\log n)^{13} .
\end{aligned}
$$

Thus,

$$
\frac{n}{(\log n)^{13}}<6 \times 10^{129}:=T \text {. }
$$

Since $T=6 \times 10^{129}>\left(4 \times 13^{2}\right)^{13}$, we can apply Lemma 2.6, to conclude that

$$
n<2^{13} T(\log T)^{13}=8 \times 10^{165} \text {. }
$$

Assume next that

$$
m \log 10=\min \{(k / 2) \log 2, m \log 10\} .
$$

Then

$$
m \log 10<10^{12} \log n .
$$

Next, we rewrite our equation as in Step 2 of Subsection 3.2, namely

$$
2^{n-2}(1+\zeta)-\left(\frac{a 10^{m}-(a-b)}{9}\right) 10^{\ell}=\frac{-b}{9},
$$

which can be rearranged as

$$
\left|2^{n-2}-\left(\frac{a 10^{m}-(a-b)}{9}\right) 10^{\ell}\right|=\left|-2^{n-2} \zeta-b / 9\right| .
$$

Dividing by $2^{n-2}$ and using Lemma 2.5, we get

$$
\begin{aligned}
\left|\left(\frac{a 10^{m}-(a-b)}{9}\right) 10^{\ell} 2^{-(n-2)}-1\right| & \leq|\zeta|+\frac{b / 9}{2^{n-2}} \\
& \leq 6 \max \left\{\frac{1}{2^{k / 2}}, \frac{1}{2^{n-2}}\right\} .
\end{aligned}
$$

The minimum of the exponents of 2 in the right-hand side is $k / 2$. Indeed, for if not, then $n-2 \leq k / 2$, so $n \leq k / 2+2<k$ since $k$ is large. However, for $n<k$, we have $F_{n}^{(k)}=2^{n-2}$, and this is not a concatenation of two repdigits by Lemma 2.7 since $n>1000$ is large. Thus, $\min \{k / 2, n-2\}=k / 2$. Let

$$
\Gamma_{4}:=\left(\frac{a 10^{m}-(a-b)}{9}\right) 10^{\ell} 2^{-(n-2)}-1 \text {. }
$$


Let us show that $\Gamma_{4} \neq 0$. Indeed, if $\Gamma_{4}=0$, we then get, by (3.15), that $2^{n-2} \zeta=-b / 9$. Thus, $F_{n}^{(k)}=2^{n-2}+2^{n-2} \zeta=2^{n-2}-b / 9$ and since this is an integer, we get that $b \in\{0,9\}$. The case $b=0$ leads to $F_{n}^{(k)}=2^{n-2}$ which we have seen that it is impossible. Thus, $b=9$ and $F_{n}^{(k)}=2^{n-2}-1$. But then,

$$
\begin{aligned}
F_{n}^{(k)} & =2^{n-2}-1=a\left(\frac{10^{m}-1}{9}\right) 10^{\ell}+9 \times\left(\frac{10^{\ell}-1}{9}\right) \\
& =a\left(\frac{10^{m}-1}{9}\right) 10^{\ell}+10^{\ell}-1,
\end{aligned}
$$

so $10^{\ell} \mid 2^{n-2}$, a contradiction. Thus, $\Gamma_{4} \neq 0$, and we can apply Matveev's Theorem 2.1 in order to get a lower bound on the left-hand side of (3.16). We take $t:=3$,

$$
\left(\gamma_{1}, \gamma_{2}, \gamma_{3}, b_{1}, b_{2}, b_{3}\right):=\left(\frac{a 10^{m}-(a-b)}{9}, 10,2,1, \ell,-(n-2)\right)
$$

A calculation similar to the one performed at $\Gamma_{2}$ in Subsection 3.2, shows that

$$
\begin{aligned}
h\left(\gamma_{1}\right) & \leq \log 9+\log \left(a 10^{m}\right)+\log (a-b)+\log 2 \leq 3 \log 9+\log 2+m \log 10 \\
& \leq 3 \log 9+\log 2+10^{12} \log n<1.1 \times 10^{12} \log n .
\end{aligned}
$$

Further, $D=1$, so we take $A_{1}:=1.1 \times 10^{12} \log n, A_{2}:=h\left(\gamma_{2}\right)=\log 10$ and $A_{3}:=h\left(\gamma_{3}\right)=\log 2$. Matveev's Theorem 2.1 tells us that

$$
\log \left|\Gamma_{4}\right|>-2.6 \times 10^{23}(1+\log n) \log n>-5 \times 10^{23}(\log n)^{2} .
$$

Comparing the above bound with (3.16) and using the fact that the minimum in the right-hand side of (3.16) occurs at $k / 2$, we get

$$
(k / 2) \log 2-\log 6<5 \times 10^{23}(\log n)^{2},
$$

so

$$
k<\frac{2}{\log 2}\left(5 \times 10^{23}(\log n)^{2}+\log 6\right)<1.5 \times 10^{24}(\log n)^{2} .
$$

By Lemma 3.2 and using again the fact that $k<n$, we get

$$
\begin{aligned}
n & <8 \times 10^{29} k^{8}(\log k)^{5} \leq 8 \times 10^{29} k^{8}(\log n)^{5} \\
& <8 \times 10^{29}\left(1.5 \times 10^{24}\right)^{8}(\log n)^{21} \\
& <2.1 \times 10^{223}(\log n)^{21} .
\end{aligned}
$$

Thus,

$$
\frac{n}{(\log n)^{21}}<2.1 \times 10^{223}:=T \text {. }
$$

By Lemma 2.6 with $m:=21$ and $T$ shown above we get

$$
n<2^{21} T(\log T)^{21}<4 \times 10^{286} .
$$

Comparing (3.17) with (3.13), we conclude that estimate (3.17) always holds. This was for $k \geq 400$. But if $k<400$, we may bound $n$ right away using the 
estimate from Lemma 3.2, and we also get that $n<10^{60}<4 \times 10^{286}$. So, in all cases, estimate (3.17) holds. We record this as a lemma.

LEMma 3.3. If $n>1000$ and $F_{n}^{(k)}$ is a concatenation of two repdigits, then

$$
n<4 \times 10^{286} .
$$

3.4. Reducing The Bound. We start with estimate (3.12).

Put

$$
\Lambda_{3}:=(m+\ell) \log 10-(n-2) \log 2+\log (a / 9) .
$$

Note that $\Gamma_{3}=e^{\Lambda_{3}}-1$. Thus, since we proved that $\Gamma_{3} \neq 0$, it follows that $\Lambda_{3} \neq 0$. Assuming $m \geq 2$, the right-hand side of (3.12) is smaller that $1 / 2$, and putting $w:=\min \{(k / 2) \log 2, m \log 10\}$, we get

$$
\left|\Lambda_{3}\right|<\frac{44}{e^{w}}
$$

Dividing both sides of the above inequality by $\log 2$, we get

$$
|(m+\ell) \tau-(n-2)+\mu|<\frac{(44 / \log 2)}{e^{w}}<\frac{A}{B^{w}},
$$

where

$$
\tau:=\frac{\log 10}{\log 2}, \quad \mu:=\frac{\log (a / 9)}{\log 2}, \quad A:=64, \quad B:=e .
$$

Furthermore, $m+\ell<M:=10^{300}$. We apply Lemma 2.2 and we take the convergent $P / Q:=p_{699} / q_{699}$ of $\tau$ which has $Q>10^{345}>6 M$. It also has $M\|Q \tau\|<10^{-45}$, while $\|Q \mu\|>0.076$ for $a \in\{1, \ldots, 8\}$, so we can take $\varepsilon:=0.076$. We get that

$$
w<\log \left(A Q \varepsilon^{-1}\right)<803 .
$$

Assume that $k>2500$. Then $(k / 2) \log 2>803$, so it must be the case that $w=m \log 10$. Thus, $m<803 / \log 10<350$. This was for $a \neq 9$, since when $a=9$ we have $\mu=0$. In this case, we have

$$
\left|\tau-\frac{n-2}{m+\ell}\right|<\frac{A}{(m+\ell) B^{w}} .
$$

Assuming $B^{w}>2 A M$, we have that the right-hand side above is smaller than $1 /\left(2(m+\ell)^{2}\right)$ so $(n-2) /(m+\ell)$ is a convergent of $\tau$. We thus conclude that $(n-2) /(m+\ell)=p_{j} / q_{j}$ for some $j \leq 699$, so $p_{j}=(n-2) / d$ and $q_{j}=(m+\ell) / d$, where we put $d:=\operatorname{gcd}(n-2, m+\ell)$. By Lemma 2.3 , the left-hand side above exceeds $1 /\left((c+2) q_{j}^{2}\right)$, where $c:=\max \left\{a_{j}: 0 \leq j \leq 699\right\}=5393$. Thus,

$$
\left.B^{w}<\frac{5395 A q_{j}}{d} \leq 5395 A(m+\ell)\right)<5395 A M,
$$

which gives

$$
w<\log (5395 A M)<704<803 .
$$


So, in both cases, namely if $a \in\{1, \ldots, 8\}$ or if $a=9$, we have that $m<350$ provided $k>2500$. We next take

$$
\Lambda_{4}:=\ell \log 10-(n-2) \log 2+\log \left(\frac{a 10^{m}-(a-b)}{9}\right) .
$$

Note that $\Gamma_{4}=e^{\Lambda_{4}}-1$. Since we proved that $\Gamma_{4} \neq 0$, it follows that $\Lambda_{4} \neq 0$. Moving to inequality (3.16), we get that the right-hand side of it is smaller than $1 / 2$. Thus,

$$
\left|\Gamma_{4}\right|<\frac{12}{2^{k / 2}}
$$

This gives

$$
\left|\ell \tau-(n-2)-\mu_{1}\right|<\frac{(12 / \log 2)}{2^{k / 2}}<\frac{A_{1}}{B_{1}^{w}}, \quad \text { where } \quad\left(A_{1}, B_{1}\right):=(18,2) .
$$

Here,

$$
\mu_{1}:=\frac{\log \left(\left(a 10^{m}-(a-b)\right) / 9\right)}{\log 2} .
$$

We take again $M:=10^{300}$ and work with the same $P / Q=p_{699} / q_{699}$. A computer calculation for $a \in\{1, \ldots, 9\}, b \in\{0, \ldots, 9\}, b \neq a$ and $m \in[1,349]$ showed that $\left\|Q \mu_{1}\right\|>0.000021$ for all $\mu_{1}$, except for the 9 triples $(a, b, m)$ :

$$
\begin{aligned}
& (1,0,1),(1,9,1),(2,0,1),(3,9,1),(4,0,1), \\
& (4,9,1),(5,0,1),(7,9,1),(8,0,1) .
\end{aligned}
$$

So, except for these 9 cases, we can take $\varepsilon:=0.00002$ and we get

$$
k / 2<\frac{\log \left(A_{1} Q \varepsilon^{-1}\right)}{\log B_{1}}<1200,
$$

so $k<2500$, a contradiction. It remains to deal with the 9 triples from (3.19). The ones having $b=0$ give

$$
F_{n}^{(k)} \in\left\{10^{\ell}, 2 \times 10^{\ell}, 4 \times 10^{\ell}, 5 \times 10^{\ell}, 8 \times 10^{\ell}\right\} .
$$

They are particular instances of numbers of the form $F_{n}^{(k)}$ whose primes factors are in the set $\{2,5\}$ and these have been found in [6]. The largest number of this form is $F_{13}^{(7)}=2000$. The other 4 cases give

$$
F_{n}^{(k)} \in\left\{2 \times 10^{\ell}-1,4 \times 10^{\ell}-1,5 \times 10^{\ell}-1,8 \times 10^{\ell}-1\right\} .
$$

For these ones, the expression $\left(a 10^{m}-(a-b)\right) / 9 \in\{2,4,5,8\}$, so that $\Gamma_{4}$ can be rewritten as

$\Lambda_{4}=(\ell+\delta) \log 5-\left(n-2-\ell-\delta_{1}\right) \log 2, \quad \delta_{1} \in\{0,1,2,3\}, \delta \in\{0,1\}, \quad \delta \delta_{1}=0$.

Thus, inequality (3.18) becomes

$$
\left|(\ell+\delta) \log 5-\left(n-2-\ell-\delta_{1}\right) \log 2\right|<\frac{12}{2^{k / 2}} .
$$


Using the continued fraction of $\log 5 / \log 2$, we get $2^{k / 2} /(12 \times 5395)<10^{300}$, which implies that $k<2500$, a contradiction since we are assuming that $k>2500$.

Thus, we showed that if $k \geq 400$, then $k \leq 2500$.

Assume that $k>500$. By Lemma 3.2 , we get $n<4 \times 10^{61}$. We repeat the same process with $M:=10^{62}$, and $P / Q=p_{139} / q_{139}$. In this case, for $\Gamma_{3}$ we have $M\|Q \tau\|<10^{-6}$, while $\|Q \mu\|>0.12$ for all $a \in\{1, \ldots, 8\}$. Thus, we can take $\varepsilon:=0.1$ and then

$$
w<\log \left(A_{1} Q \varepsilon^{-1}\right)<164 .
$$

If $k>500$, then $(k / 2) \log 2>173$, so we must have $w=m \log 10<164$, so $m<72$. If $a=9$, then by the previous argument, we get

$$
B_{1}^{w}<5395 A_{1} M, \quad \text { which gives } w<156 .
$$

Since $k>500$, we get $m<68$. Thus, $m<72$ regardless of the value of a. Now we move to $\Gamma_{4}$. Working with the same $M$ and $P / Q$ and ignoring the 9 triples shown at (3.19), we get that $\left\|Q \mu_{1}\right\|>0.000041$, so we can take $\varepsilon:=0.0004$. Thus,

$$
(k / 2) \log 2<173,
$$

which contradicts the fact that $k>500$. In the case when $(a, b, m)$ is one of the 9 triples shown at $(3.19)$, we get $B_{1}^{w}<5395 A_{1} M$, so $(k / 2) \log 2<155$, which contradicts again the fact that $k>500$.

Thus, $k \leq 500$. By Lemma 3.2, we get $n<3 \times 10^{55}$. So, we take $M:=10^{56}$ and we work with

$$
\Lambda_{1}:=(m+\ell) \log 10-(n-1) \log \alpha-\log \left(9 f_{k}(\alpha) / a\right) .
$$

Assuming $m \geq 2$, the right-hand side of $(3.3)$ is $<1 / 2$. Since $\Gamma_{1}=e^{-\Lambda_{1}}-1$, we get that

$$
\left|\Lambda_{1}\right|<\frac{30}{10^{m}}
$$

This can be rewritten as

$$
\left|(m+\ell) \tau_{k}-(n-1)-\nu_{k}\right|<\frac{30 / \log \alpha_{k}}{10^{m}}<\frac{A_{2}}{B_{2}}
$$

where we can take

$$
\tau_{k}:=\frac{\log 10}{\log \alpha}, \quad \nu_{k}:=\frac{\log \left(9 f_{k}(\alpha) / a\right)}{\log \alpha}, \quad A_{2}:=63, \quad B_{2}:=10 .
$$

We take $P / Q=p_{279} / q_{279}$ for all $k \in[2,500]$. The maximum $M\left\|Q \tau_{k}\right\|$ is smaller than $10^{-68}$. The minimum $\left\|Q \mu_{k}\right\|$ is larger than $5 \times 10^{-11}$. The maximum $Q$ satisfies $Q<10^{160}$. So, we can take $\varepsilon:=10^{-11}$ for all choices of $k \in[2,500]$ and $a \in[1,9]$, getting

$$
m<\frac{\log \left(A_{2} Q \varepsilon^{-1}\right)}{\log 10}<173 .
$$


We next move to

$$
\Gamma_{2}:=\ell \log 10-(n-1) \log \alpha-\log \left(\frac{\left.a 10^{m}-(a-b)\right)}{9 f_{k}(\alpha)}\right) .
$$

Since $n>1000$, the right-hand side of (3.6) is $<1 / 2$. Since $\Gamma_{2}=e^{\Lambda_{2}}-1$, we get that

$$
\left|\Lambda_{2}\right|<\frac{6}{\alpha^{n-1}} .
$$

Since $\Gamma_{2} \neq 0$, it follows that $\Lambda_{2} \neq 0$. It then follows that

$$
\left|\ell \tau_{k}-(n-1)-(n-1)-\mu_{k}^{\prime}\right|<\frac{(6 / \log \alpha)}{\alpha^{n-1}}<\frac{A_{3}}{B_{3}},
$$

where

$$
\mu_{k}^{\prime}:=\frac{\left.\log \left(a 10^{m}-(a-b)\right) / 9 f_{k}(\alpha)\right)}{\log \alpha}, \quad \text { and } \quad\left(A_{3}, B_{3}\right):=(13, \alpha)
$$

We kept the same $M$ and the same $P / Q$. We got that $Q \varepsilon^{-1}<10^{170}$ for all $k \in[2,500], a \in\{1, \ldots, 9\}, b \in\{0, \ldots, 9\}, b \neq a$, and $m \in[1,174]$. Hence,

$$
n-1<\frac{\log \left(A_{3} Q \varepsilon^{-1}\right)}{\log \alpha}<820,
$$

which is the final contradiction. Thus, Theorem 1.1 is proved.

ACKNOWLEDGEMENTS.

We thank the referees for a careful reading of our manuscript and for useful comments and remarks.

\section{REFERENCES}

[1] A. Alahmadi, A. Altassan, F. Luca and H. Shoaib, Fibonacci numbers which are concatenations of two repdigits, Quaestiones Math. (2019), 1-10.

[2] S. D. Alvarado and F. Luca, Fibonacci numbers which are sums of two repdigits, Proceedings of the XIVth International Conference on Fibonacci numbers and their applications, Sociedad Matematica Mexicana, Aportaciones Matemáticas, Investigación 20 (2011), 97-108.

[3] W. D. Banks and F. Luca, Concatenations with binary recurrent sequences, J. Integer Seq. 8 (2005), Article 05.1.13.

[4] A. Baker and G. Wüstholz, Logarithmic forms and group varieties, J. Reine Angew. Math. 442 (1993), 19-62.

[5] J. J. Bravo and F. Luca, On a conjecture about repdigits in $k$-generalized Fibonacci sequences, Publ. Math. Debrecen 82 (2013), 623-639.

[6] J. J. Bravo and F. Luca, On the largest prime factor of the $k$-Fibonacci numbers, Internat. J. Number Theory 9 (2013), 1351-1366.

[7] Y. Bugeaud, M. Maurice and S. Siksek, Classical and modular approaches to exponential Diophantine equations I. Fibonacci and Lucas perfect powers, Ann. of Math. 163 (2006), 969-1018.

[8] Y. Bugeaud and M. Mignotte, On integers with identical digits, Mathematika 46 (1999), 411-417.

[9] M. Ddamulira and F. Luca, On the problem of Pillai with $k$-generalized Fibonacci numbers and powers of 3, Int. J. Number Theory 16 (2020), 1643-1666. 
[10] G. P. Dresden and Z. Du, A simplified Binet formula for $r$-generalized Fibonacci numbers, J. Integer Seq. 17 (2014), Article 14.4.7.

[11] A. Dujella and A. Pethö, A generalization of a theorem of Baker and Davenport, Quart. J. Math. Oxford Ser. (2) 49 (1998), 291-306.

[12] M. R. Murty and J. Esmonde, Problems in algebraic number theory, Second edition. Graduate Texts in Mathematics 190, Springer-Verlag, New York, 2005.

[13] C. A. Gómez and F. Luca, On the distribution of the roots of $x^{k}-x^{k-1}-\cdots-x-1$, Comment. Math. Univ. Carolinae, to appear.

[14] S. Guzmán-Sanchez and F. Luca, Linear combinations of factorials and S-units in a binary recurrence sequence, Ann. Math. Québec 38 (2014), 169-188.

[15] F. Luca, Fibonacci and Lucas numbers with only one distinct digit, Port. Math. 57 (2000), 243-254.

[16] F. Luca, Repdigits as sums of three Fibonacci numbers, Math. Comm. 17 (2012), $1-11$.

[17] D. Marques and A. Togbé, On terms of linear recurrence sequences with only one distinct block of digits, Colloq. Math. 124 (2011), 145-155.

[18] D. Marques and A. Togbé, On repdigits as product of consecutive Fibonacci numbers, Rend. Instit. Mat. Univ. Trieste 44 (2012), 393-397.

[19] E. M. Matveev, An explicit lower bound for a homogeneous rational linear form in logarithms of algebraic numbers. II, Izv. Ross. Akad. Nauk Ser. Mat. 64 (2000), 125180. English translation in Izv. Math. 64 (2000), 1217-1269.

[20] R. Obláth, Une propriété des puissances parfaites, Mathesis 65 (1956), 356-364.

A. Alahmadi

Research Group in Algebraic Structures and Applications

King Abdulaziz University, Jeddah, Saudi Arabia

E-mail: analahmadi@kau.edu.sa

A. Altassan

Research Group in Algebraic Structures and Applications

King Abdulaziz University, Jeddah, Saudi Arabia

E-mail: aaltassan@kau.edu.sa

F. Luca

School of Mathematics

Wits University, Johannesburg, South Africa

$\&$

Research Group in Algebraic Structures and Applications

King Abdulaziz University, Jeddah, Saudi Arabia

\&

Centro de Ciencias Matemáticas

UNAM, Morelia, Mexico

E-mail: florian.luca@its.ac.za

H. Shoaib

Research Group in Algebraic Structures and Applications

King Abdulaziz University, Jeddah, Saudi Arabia

E-mail: hashoaib@kau.edu.sa

Received: 14.3 .2020 .

Revised: 22.7.2020. 